\title{
Hard X-Ray Scanning Microscopy with Coherent Radiation: Beyond the Resolution of Conventional X-Ray Microscopes
}

\author{
A. Schropp ${ }^{1}$, R. Hoppe ${ }^{1}$, J. Patommel ${ }^{1}$, D. Samberg ${ }^{1}$, F. Seiboth ${ }^{1}$, \\ S. Stephan ${ }^{1}$, G. Wellenreuther ${ }^{2}$, G. Falkenberg ${ }^{2}$, and C. G. Schroer ${ }^{1, a}$ ) \\ ${ }^{1}$ Institute of Structural Physics, Technische Universität Dresden, D-01062 Dresden, Germany \\ ${ }^{2}$ Deutsches Elektronen Synchrotron DESY, D-22607 Hamburg, Germany
}

(Dated: October 1, 2013)

\begin{abstract}
We demonstrate $\mathrm{x}$-ray scanning coherent diffraction microscopy (ptychography) with $10 \mathrm{~nm}$ spatial resolution, clearly exceeding the resolution limits of conventional hard x-ray microscopy. The spatial resolution in a ptychogram is shown to depend on the shape (structure factor) of a feature and can vary for different features in the object. In addition, the resolution and contrast is shown to increase with increasing coherent fluence. For an optimal ptychographic x-ray microscope this implies a source with highest possible brilliance and ultimately an $\mathrm{x}$-ray optic with a large numerical aperture to generate the optimal probe beam.
\end{abstract}

In order to understand the function of nanoscopic objects and materials, such as organelles in biological cells, nano electronic devices, or nano structured materials such as catalysts, it is crucial to be able to determine their structure. Ideally, this is done in the natural environment of the specimen or under working conditions, e. g., the investigation of electromigration in a via of a functioning electronic device ${ }^{1}$ or the activity of small catalyst particles inside a chemical reactor. ${ }^{2}$ Hard $\mathrm{x}$-ray microscopy is ideal to address these questions, as the large penetration depth of hard x-rays in matter allows one to investigate the inner structures of an object without destructive sample preparation or in-situ inside of special sample environments. ${ }^{3}$

In conventional $\mathrm{x}$-ray microscopy the spatial resolution is currently limited to a few $10 \mathrm{~nm},{ }^{3}$ mainly due to aberrations and the limited numerical aperture of today's x-ray optics. ${ }^{4-7}$ By combining scanning microscopy with coherent $\mathrm{x}$-ray diffraction imaging this limit can be overcome. In recent years, scanning coherent diffraction microscopy, also known as ptychography, has been rediscovered ${ }^{8}$ and introduced into the field of x-ray imaging. ${ }^{9}$ In ptychography, the sample is scanned through a confined coherent beam with appropriate overlap between the illuminated areas of neighboring scan points. At each position of the scan, a far-field diffraction pattern is recorded. The diffraction patterns are then fed into a phasing algorithm to reconstruct the object's transmission function and the wave field of the illumination. ${ }^{10}$ The simultaneous reconstruction of the illumination is quite important as the illuminating beam is typically not known very well. As a result, ptychography has revolutionized the characterization of x-ray optics. ${ }^{6,11}$ Ptychographic imaging has been applied to real samples ${ }^{12}$ and extended to three dimensions by a combination with tomography. ${ }^{13}$

In this Letter, we demonstrate that ptychographic imaging yields spatial resolutions well beyond the state of art of conventional $\mathrm{x}$-ray microscopy and discuss the physical limitations and the resulting requirements on ptychographic x-ray microscopes. Mechanical and x-ray optical stability is one crucial requirement, ${ }^{14}$ but the main limitation is given by the strength of the scattering signal in the diffraction patterns. For a given feature in an object, the resolution and contrast de- pends on its scattering strength (structure factor) and on the fluence, i. e., the number of photons per unit area, applied to the feature. ${ }^{15}$ This is illustrated by high-resolution images obtained by coherent x-ray diffraction microscopy with focused synchrotron radiation. ${ }^{16}$

To build an efficient ptychographic microscope, the coherent fluence needs to be optimized. For a fixed field of view of area $A$, the fluence is only determined by the coherent flux $F_{\mathrm{c}}$ of the source and the exposure time. As $F_{\mathrm{c}}$ is directly related to the brilliance $\mathrm{Br}$ of the source, optimal performance will be reached at the most brilliant sources. ${ }^{17}$ The spatial resolution can, however, be further increased if the field of view is reduced, condensing the coherent flux onto a smaller area and increasing the coherent fluence together with the coherent intensity $I_{\mathrm{c}}$ on the sample. Optimal focusing of the coherent flux $F_{\mathrm{c}}$ is obtained by diffraction limited optics with high numerical aperture $N A$ :

$$
I_{\mathrm{c}}=\frac{F_{\mathrm{c}}}{A_{\mathrm{eff}}} \propto B r \times \frac{\Delta E}{E} \times N A^{2} \times T .
$$

Here, $A_{\text {eff }} \propto(\lambda / N A)^{2}$ is the effective area of the diffraction limited focus, $\Delta E / E$ is the energy bandwidth, and $T$ is the efficiency of the focusing optic. An optimal ptychographic microscope is thus placed at the most brilliant x-ray source and ultimately makes use of optics with highest numerical aperture. ${ }^{18}$ At the same time, such a scanning microscope yields the best conventional x-ray scanning micrographs.

We have constructed and built a hard x-ray scanning microscope at beamline P06 of the new low-emittance synchrotron radiation source PETRA III at DESY in Hamburg. ${ }^{19}$ It is installed at a distance of approximately $98 \mathrm{~m}$ from the undulator source. On the way to the instrument, the x-ray beam is monochromatized by a $\mathrm{Si}$ (111) double-crystal monochromator, and higher harmonics are reduced by a pair of total reflection mirrors. In addition, the lateral coherence length at the instrument can be adjusted by prefocusing optics. ${ }^{19}$ Inside the scanning microscope, the beam is usually focused by a pair of crossed nanofocusing refractive $x$-ray lenses made of silicon ${ }^{4,20}$ but other optics, such as Fresnel zone plates ${ }^{6}$ and multilayer Laue lenses ${ }^{5}$ can be used, as well. By means of x-ray fluorescence, absorption, and (coherent) scattering, 


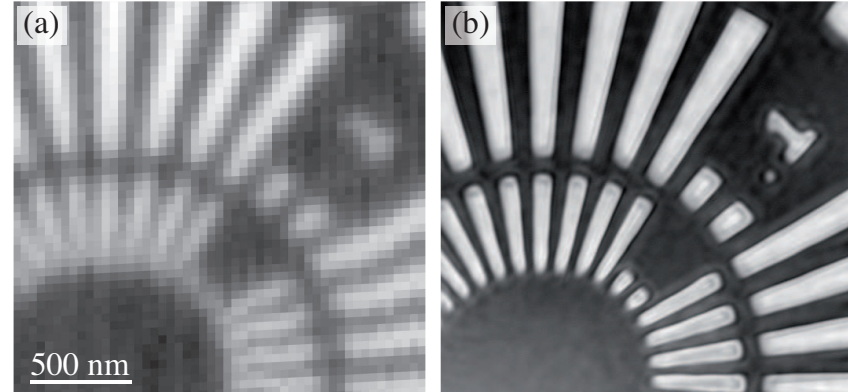

FIG. 1: (a) Fluorescence map ( $\mathrm{Ta} \mathrm{K}_{\alpha}$ ) of an NTT-AT resolution test chart. The spatial resolution is given by the full width at half maximum $\left(\mathrm{FWHM}\right.$ ) beam size of the probe to $80 \times 80 \mathrm{~nm}^{2}$ (pixel size $40 \mathrm{~nm}$ ). (b) Corresponding reconstructed ptychographic image showing a significantly improved spatial resolution (pixel size $3.84 \mathrm{~nm})$.

this scanning microscope can image specimens with elemental, chemical, and structural contrast, respectively. In conventional scanning mode, spatial resolutions of $80 \mathrm{~nm}$ are obtained routinely, and beam sizes down to $25 \mathrm{~nm}$ have been reached.

Fig. 1(a) shows the fluorescence map of a resolution test chart by NTT-AT (model: ATN/XRESO-50HC) made of tantalum (material thickness $500 \mathrm{~nm}$ ). The smallest features (50 $\mathrm{nm}$ lines and spaces) are clearly resolved with a $80 \times 80 \mathrm{~nm}^{2}$ diffraction limited beam generated with nanofocusing refractive lenses at $15.25 \mathrm{keV}$. Fig. 1(b) shows the reconstructed ptychogram that was recorded simultaneously with the fluorescence map. For the images shown in Fig. 1, an area of $2 \times 2 \mu \mathrm{m}^{2}$ was scanned with a focused coherent $\mathrm{x}$-ray beam with a step size of $40 \mathrm{~nm}(50 \times 50$ steps $)$. The exposure time was $1.5 \mathrm{~s}$ per scan point, applying a detected fluence of $2.75 \times 10^{4} \mathrm{ph} / \mathrm{nm}^{2}$.

A comparison of Fig. 1(a) and (b) shows clearly that ptychographic imaging can significantly enhance the spatial resolution over that given in conventional scanning microscopy. To analyse the resolution in more detail, the ptychographic reconstruction is enlarged in Fig. 2(a), and several line scans [Fig. 2(b)] are evaluated by fitting an error function to them. The straight sharp edges in the test pattern are imaged with a resolution of about $10 \mathrm{~nm}$. This exceeds that of the best conventional hard x-ray microscopes available today. These features have the largest structure factor along a streak perpendicular to the edge up to high momentum transfer $q$ and are thus reconstructed with highest spatial resolution. ${ }^{21}$ Fig. 2(d) shows the diffraction pattern recorded at the edge near line scan 1 . The small dot analyzed by line scan 3 in Fig. 2(a) scatters isotropically in all directions as illustrated by the diffraction pattern in Fig. 2(c) that was recorded on the dot during the ptychographic scan. Thus its structure factor decays quicker with increasing momentum transfer $q$, and its spatial resolution is lower. ${ }^{22} \mathrm{~A}$ similar reduction in spatial resolution is also seen for a round feature at the tip of the spoke in line scan 4 in Fig. 2.

In conclusion from the previous analysis the spatial reso-
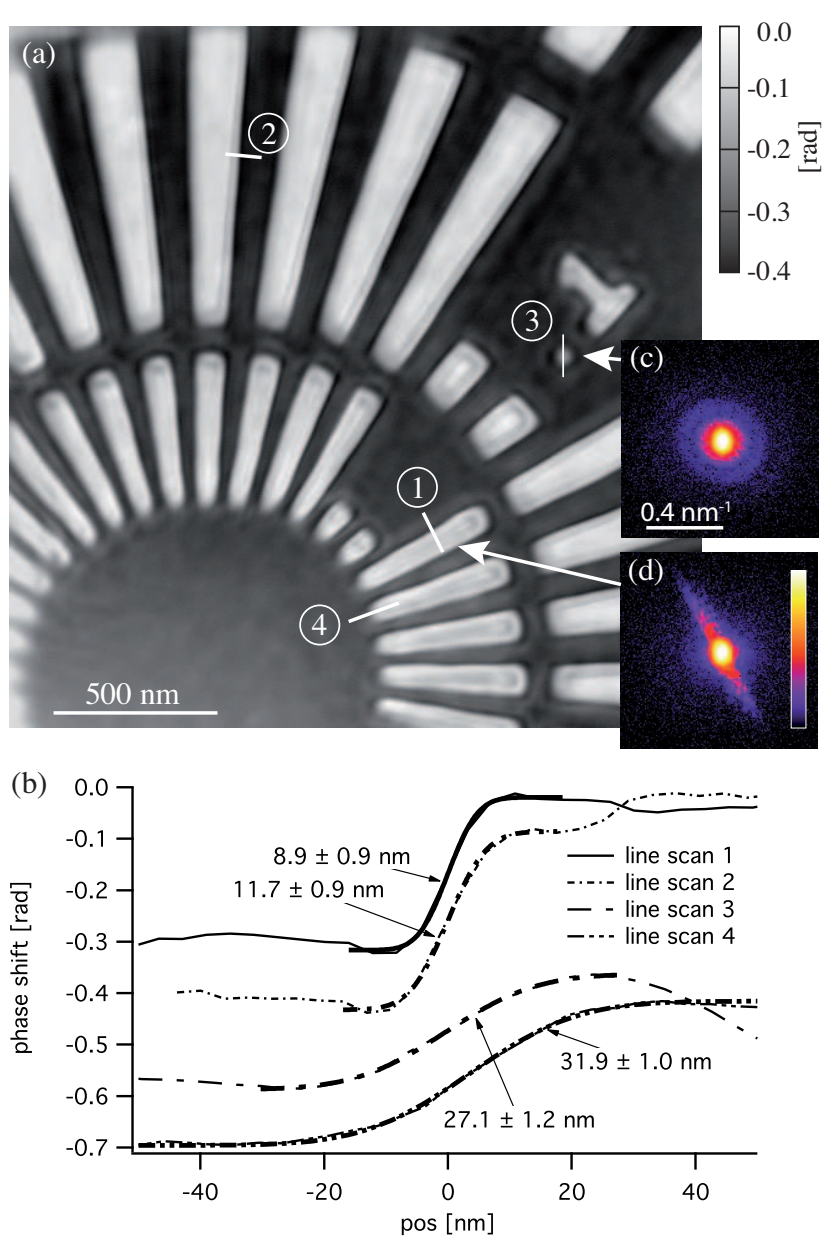

FIG. 2: (color online) (a) Enlarged view of the ptychographic reconstruction of the resolution test pattern shown in Fig. 1(b) (quantitative phase map). (b) Line spread measured at different positions in (a). An error function is fitted to the line scans, and the FWHM spread is given for each. (c) Far-field diffraction pattern recorded at the position of the dot during the ptychographic scan, (d) similar diffraction pattern on the edge near line scan 1 (both on logarithmic scale). Line scan 3 and 4 are shifted by $-0.2 \mathrm{rad}$ and $-0.4 \mathrm{rad}$ for better presentation, respectively.

lution and contrast of different features in a ptychogram can vary. It will, however, improve for all features with a growing fluence on the sample. This is illustrated in Fig. 3 by two ptychograms recorded of a front-end processed passivated microchip. Fig. 3(a) shows an area of $2 \times 2 \mu \mathrm{m}^{2}$ and was recently recorded at the nanoprobe station P06 at PETRA III, scanning the sample with similar parameters as the previous test pattern, except for a slighty shorter exposure time of $1 \mathrm{~s}$. As the microchip is on a silicon substrate that has to be penetrated by the x-rays, the detected fluence is reduced to $6.7 \times 10^{3} \mathrm{ph} / \mathrm{nm}^{2}$. For comparison, Fig. 3(b) shows an earlier $\mathrm{x}$-ray ptychogram of the microchip that was recorded with significantly less fluence, i. e., $3.9 \times 10^{2} \mathrm{ph} / \mathrm{nm}^{2} .{ }^{23} \mathrm{Al}-$ though a direct comparison is difficult as both images in Fig. 3 do not show the same detail of the microchip, the far superior 


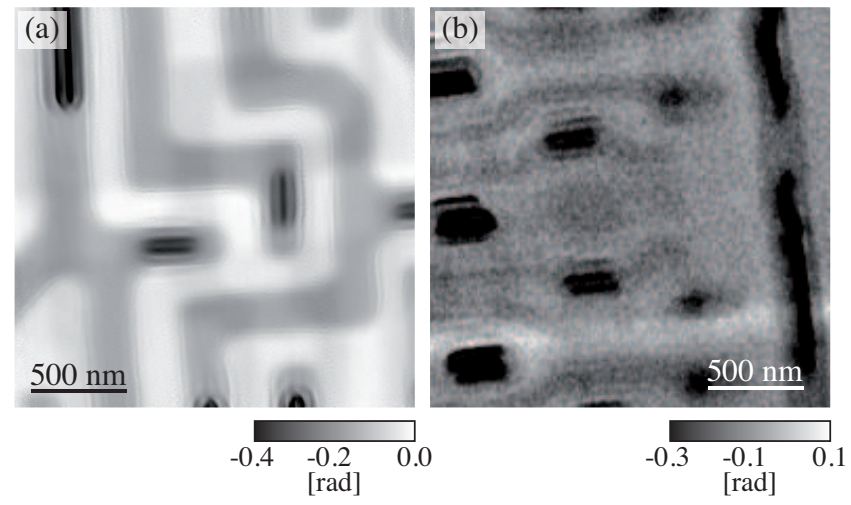

FIG. 3: Ptychographic images a front-end processed and passivated microchip (cf. Ref. 15). (a) Ptychogram recorded with a detected fluence of $6.7 \times 10^{3} \mathrm{ph} / \mathrm{nm}^{2}$, (b) ptychogram of another field of view (cf. Ref. 23, detected fluence: $3.9 \times 10^{2} \mathrm{ph} / \mathrm{nm}^{2}$ ). In (b), the gray scale is slightly shifted to enhance the contrast.

signal-to-noise ratio in (a) as compared to (b) becomes apparent. In Fig. 3(b) the phase sensitivity is about $20 \mathrm{mrad}$, and only the features with the strongest contrast can be seen, i. e., tungsten plugs and vias. ${ }^{12}$ In Fig. 3(a) the phase sensitivity is enhanced to about $1 \mathrm{mrad}$, revealing structures in other layers of the chip with weaker contrast compared to the tungsten plugs and vias. The factor 17 in fluence also leads to an in- crease in spatial resolution by about a factor 2 that is expected for an approximate power-law behavior of $q^{-\alpha}(\alpha \approx 4)$ of the intensity in the diffraction patterns.

The spatial resolution that can be obtained in a ptychogram mainly depends on the structure factor of the features in the object and on the fluence on the sample. This assumes that technical issues, such as mechanical instabilities and background scattering signals, are minimized. To improve spatial resolution well below $10 \mathrm{~nm}$, the coherent flux density in the microscope needs to be increased. For the nanoprobe station at beamline P06 this can be achieved by better focusing the coherent flux to the aperture of the nanofocusing optics (aperture matching) and by improving their numerical aperture. In this way, up to three orders of magnitude in flux density can still be gained, increasing the spatial resolution by almost one order of magnitude. In the future, spatial resolutions on the scale of $1 \mathrm{~nm}$ should be reachable in this way for radiation hard and strongly scattering objects. Instrumentally, the stability of the microscope will have to be improved. From a methodological point of view algorithms are required that properly model the partial coherence of aperture-matched nanobeams.

This work is supported by the German Ministry of Education and Research (BMBF) under grant number 05K10OD1 and by VH-VI-203 and VH-VI-403 of the Impuls- und Vernetzungsfonds (IVF) of the Helmholtz Association of German Research Centres.
${ }^{1}$ E. Zschech, R. Huebner, D. Chumakov, O. Aubel, D. Friedrich, P. Guttmann, S. Heim, and G. Schneider, J. Appl. Phys. 106, 093711 (2009).

2 J.-D. Grunwaldt and C. G. Schroer, Chem. Soc. Rev. 39, 4741 (2010).

3 Proceedings of the 10th International Conference on X-Ray Microscopy, AIP Conference Series Vol. 1365, edited by I. McNulty, C. Eyberger, and B. Lai (AIP, Melville, 2011).

${ }^{4}$ C. G. Schroer, O. Kurapova, J. Patommel, P. Boye, J. Feldkamp, B. Lengeler, M. Burghammer, C. Riekel, L. Vincze, A. van der Hart, and M. Küchler, Appl. Phys. Lett. 87, 124103 (2005).

5 H. C. Kang, J. Maser, G. B. Stephenson, C. Liu, R. Conley, A. T. Macrander, and S. Vogt, Phys. Rev. Lett. 96, 127401 (2006).

6 J. Vila-Comamala, A. Diaz, M. Guizar-Sicairos, A. Mantion, C. M. Kewish, A. Menzel, O. Bunk, and C. David, Opt. Express 19, 21333 (2011).

7 O. Hignette, P. Cloetens, G. Rostaing, P. Bernard, and C. Morawe, Rev. Sci. Instrum. 76, 063709 (2005); Y. S. Chu, J. M. Yi, F. De Carlo, Q. Shen, W.-K. Lee, H. J. Wu, C. L. Wang, J. Y. Wang, C. J. Liu, C. H. Wang, S. R. Wu, C. C. Chien, Y. Hwu, A. Tkachuk, W. Yun, M. Feser, K. S. Liang, C. S. Yang, J. H. Je, and G. Margaritondo, Appl. Phys. Lett. 92, 103119 (2008); H. Mimura, S. Handa, T. Kimura, H. Yumoto, D. Yamakawa, H. Yokoyama, S. Matsuyama, K. Inagaki, K. Yamamura, Y. Sano, K. Tamasaku, Y. Nishino, M. Yabashi, T. Ishikawa, and K. Yamauchi, Nature Physics 6, 122 (2010).

8 J. M. Rodenburg and H. M. L. Faulkner, Appl. Phys. Lett. 85, 4795 (2004); H. M. L. Faulkner and J. M. Rodenburg, Phys. Rev. Lett. 93, 023903 (2004).
9 J. M. Rodenburg, A. C. Hurst, A. G. Cullis, B. R. Dobsen, F. Pfeiffer, O. Bunk, C. David, K. Jefimovs, and I. Johnson, Phys. Rev. Lett. 98, 034801 (2007); P. Thibault, M. Dierolf, A. Menzel, O. Bunk, C. David, and F. Pfeiffer, Science 321, 379 (2008).

${ }^{10}$ P. Thibault, M. Dierolf, O. Bunk, A. Menzel, and F. Pfeiffer, Ultramicroscopy 109, 338 (2009); A. M. Maiden and J. M. Rodenburg, Ultramicroscopy 109, 1256 (2009).

11 A. Schropp, P. Boye, J. M. Feldkamp, R. Hoppe, J. Patommel, D. Samberg, S. Stephan, K. Giewekemeyer, R. N. Wilke, T. Salditt, J. Gulden, A. P. Mancuso, I. A. Vartanyants, E. Weckert, S. Schöder, M. Burghammer, and C. G. Schroer, Appl. Phys. Lett. 96, 091102 (2010); C. M. Kewish, M. Guizar-Sicairos, C. Liu, J. Qian, B. Shi, C. Benson, A. M. Khounsary, J. Vila-Comamala, O. Bunk, J. R. Fienup, A. T. Macrander, and L. Assoufid, Opt. Express 18, 23420 (2010); C. M. Kewish, P. Thibault, M. Dierolf, O. Bunk, A. Menzel, J. Vila-Comamala, K. Jefimovs, and F. Pfeiffer, Ultramicroscopy 110, 325 (2010); S. Hönig, R. Hoppe, J. Patommel, A. Schropp, S. Stephan, S. Schöder, M. Burghammer, and C. G. Schroer, Opt. Express 19, 16325 (2011).

12 A. Schropp, P. Boye, A. Goldschmidt, S. Hönig, R. Hoppe, J. Patommel, C. Rakete, D. Samberg, S. Stephan, S. Schöder, M. Burghammer, and C. G. Schroer, J. Microscopy 241, 9 (2011).

${ }_{13}$ M. Dierolf, A. Menzel, P. Thibault, P. Schneider, C. M. Kewish, R. Wepf, O. Bunk, and F. Pfeiffer, Nature 467, 436 (2010); A. Diaz, P. Trtik, M. Guizar-Sicairos, A. Menzel, P. Thibault, and O. Bunk, Phys. Rev. B 85, 020104(R) (2012).

${ }^{14}$ Y. Takahashi, A. Suzuki, N. Zettsu, Y. Kohmura, Y. Senba, H. Ohashi, K. Yamauchi, and T. Ishikawa, Phys. Rev. B 83, 214109 (2011). 
15 A. Schropp and C. G. Schroer, New Journal of Physics 12, 035016 (2010).

16 C. G. Schroer, P. Boye, J. Feldkamp, J. Patommel, A. Schropp, A. Schwab, S. Stephan, M. Burghammer, S. Schöder, and C. Riekel, Phys. Rev. Lett. 101, 090801 (2008); Y. Takahashi, Y. Nishino, R. Tsutsumi, H. Kubo, H. Furukawa, H. Mimura, S. Matsuyama, N. Zettsu, E. Matsubara, T. Ishikawa, and K. Yamauchi, Phys. Rev. B 80, 054103 (2009).

${ }^{17} F_{\mathrm{c}} \propto B r \times \lambda^{2} \times \Delta E / E$, where $\lambda$ is the wavelength of the x-rays and $\Delta E / E$ their energy bandwidth.

${ }^{18}$ Compared to ptychographic imaging without focusing, more than six orders of magnitude in fluence can be gained in this way, improving the spatial resolution by one and a half to two orders of magnitude.

${ }^{19}$ C. G. Schroer, P. Boye, J. M. Feldkamp, J. Patommel, D. Samberg, A. Schropp, A. Schwab, S. Stephan, G. Falkenberg, G. Wellenreuther, and N. Reimers, Nucl. Instrum. Meth. A 616, 93 (2010).
${ }^{20}$ C. G. Schroer, M. Kuhlmann, U. T. Hunger, T. F. Günzler, O. Kurapova, S. Feste, F. Frehse, B. Lengeler, M. Drakopoulos, A. Somogyi, A. S. Simionovici, A. Snigirev, I. Snigireva, C. Schug, and W. H. Schröder, Appl. Phys. Lett. 82, 1485 (2003).

${ }^{21}$ Sharp edges introduce sharp streaks in the diffraction pattern [Fig. 2(a)], yielding high resolution information in the direction perpendicular to the edge. As a result, the edge is reconstructed sharply and smoothly. In order to see small features on the edge, e. g., details of its roughness, their contribution to the diffraction needs to be detected, requiring good statistics also in the low intensity parts of the diffraction pattern [Fig. 2(a)]. ${ }^{15}$ This will require an increased fluence on the sample.

22 This argument ${ }^{15}$ is independent of a feature being illuminated by itself or together with others in a larger field of illumination.

${ }^{23}$ Fig. 3(b) is a $2 \times 2 \mu \mathrm{m}^{2}$ detail of the micrograph shown in Schropp et al., ${ }^{12}$ Fig. 1. 